\title{
LC/MS-Based Global Metabolomic Identification of Serum Biomarkers Differentiating Hepatocellular Carcinoma from Chronic Hepatitis B and Liver Cirrhosis
}

\author{
Hong Y. Pan," Qing Q. Wu," Qiao Q. Yin, Yi N. Dai, Yi C. Huang, Wei Zheng, Tian C. Hui," \\ Mei J. Chen, Ming S. Wang, Jia J. Zhang, Hai J. Huang, ${ }^{*}$ and Yong X. Tong*
}

Cite This: ACS Omega 2021, 6, 1160-1170

Read Online

ABSTRACT: Chronic hepatitis B virus ( $\mathrm{CHB}$ ) infection is one of the primary risk factors associated with the development of hepatocellular carcinoma (HCC). Despite having been extensively studied, diagnosing early-stage HCC remains challenging, and diagnosed patients have a poor (3-5\%) survival rate. Identifying new approaches to detect changes in the serum metabolic profiles of patients with $\mathrm{CHB}$ and liver cirrhosis (LC) may provide a valuable approach to better detect HCC at an early stage when it is still amenable to treatment, thereby improving patient prognosis and survival. In the present study, we, therefore, employed a liquid chromatography-mass spectrometry (LC-MS)-based approach to evaluate the serum metabolic profiles of $30 \mathrm{CHB}$ patients, $29 \mathrm{LC}$ patients, and $30 \mathrm{HCC}$ patients. We then employed appropriate statistical methods to identify those metabolites that were best able to distinguish HCC cases from LC and CHB controls. A mass-based database was then used to putatively identify these metabolites. We then confirmed the identities of a subset of

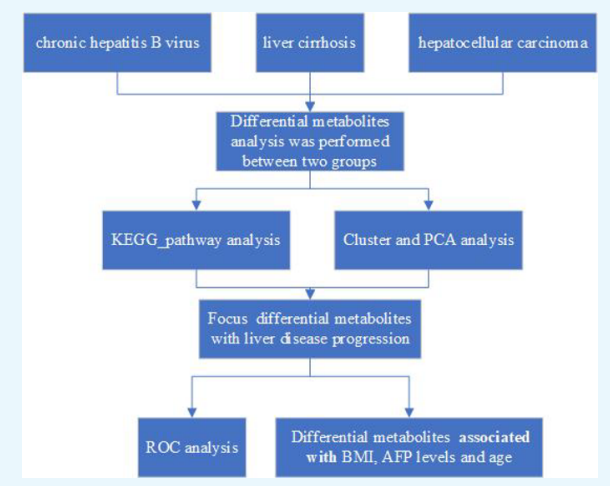
these metabolites through comparisons with the MS/MS fragmentation patterns and retention times of reference standards. The serum samples were then reanalyzed to quantify the levels of these selected metabolites and of other metabolites that have previously been identified as potential HCC biomarkers. Through this approach, we observed clear differences in the metabolite profiles of the $\mathrm{CHB}, \mathrm{LC}$, and HCC patient groups in both positive- and negative-ion modes. We found that the levels of taurodeoxy cholic acid (TCA) and 1,2-diacyl-3- $\beta$-D-galactosyl-sn-glycerol rose with the progression from CHB to LC to HCC, whereas levels of 5-hydroxy$6 E, 8 Z, 11 Z, 14 Z, 17 Z$-eicosapentaenoic acid, and glycyrrhizic acid were gradually reduced with liver disease progression in these groups. The ROC analysis showed that taurodeoxy cholic acid (TCA), 1,2-diacyl-3- $\beta$-D-galactosyl-sn-glycerol, 5-hydroxy$6 E, 8 Z, 11 Z, 14 Z, 17 Z$-eicosapentaenoic acid, and glycyrrhizic acid had a diagnosis performance with liver disease progression. These four metabolites have a significant correlation with alpha fetal protein (AFP) level and age. Our results highlight novel metabolic biomarkers that have the potential to be used for differentiating between CHB, LC, and HCC patients, thereby facilitating the identification and treatment of patients with early-stage HCC.

\section{INTRODUCTION}

Hepatocellular carcinoma (HCC) remains one of the most prevalent forms of cancer and the third leading cause of cancerassociated mortality with a 5 -year survival rate of $<7 \%$. ${ }^{1,2}$ One of the primary risk factors for HCC development is chronic hepatitis $\mathrm{B}$ virus $(\mathrm{CHB})$ infection, which can progress to liver cirrhosis (LC) and HCC. ${ }^{3,4}$ In high-risk populations such as LC patients, it is essential that HCC is detected at an early stage when the treatments still have the potential to cure disease or to significantly prolong patient survival. ${ }^{5}$ Currently, the majority of HCC patients are not diagnosed until the disease is in an advanced stage owing to a lack of symptoms associated with a precancerous state and early stages of oncogenesis. ${ }^{6}$ The most sensitive clinical biomarker of HCC at present is alpha fetal protein (AFP), which is still of limited utility given that it has a disappointing sensitivity of just $70 \%{ }^{7}$ It is thus essential that a novel approach to identifying HCC- specific biomarkers be identified to facilitate early-stage HCC detection, diagnosis, and treatment so as to improve patient prognosis.

Metabolomic studies offer a comprehensive approach to characterize the biochemical profiles of particular physiological or pathological states. Such metabolomic approaches thus have the potential to offer valuable insights into the molecular mechanisms governing HCC development and progression., Liquid chromatography-mass spectrometry (LC-MS) ap-

Received: September 1, 2020

Accepted: December 7, 2020

Published: January 4, 2021 


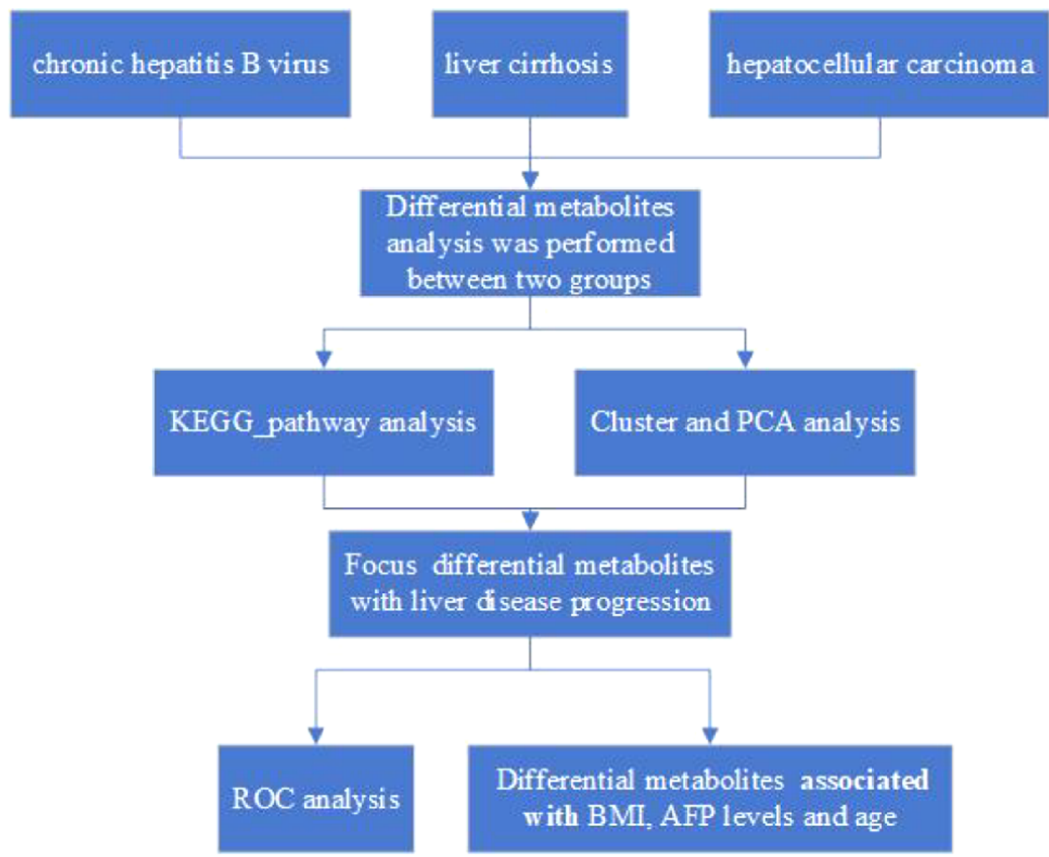

Figure 1. Schedule of the research.

Table 1. Summary of Patient Demographics and Characteristics

\begin{tabular}{llll} 
& CHB $(N=27)$ & LC $(N=29)$ & HCC $(N=25)$ \\
clinical_NA & 3 & 1 & 4 \\
age (years), mean \pm SD & $41.67 \pm 10.99$ & $51.62 \pm 9.5$ & $60.6 \pm 8.97$ \\
AFP (ng/mL), mean \pm SD & $4.31 \pm 4.2$ & $15.76 \pm 67.98$ & $7990.88 \pm 24565.75$ \\
height (cm), mean \pm SD & $170.74 \pm 7.39$ & $169.62 \pm 7.41$ & $170.48 \pm 5.88$ \\
weight (kg), mean \pm SD & $67.06 \pm 7.64$ & $65.3 \pm 9.26$ & $68.36 \pm 8.74$ \\
BMI, mean \pm SD & $23 \pm 2.18$ & $22.59 \pm 1.99$ & $23.49 \pm 2.38$ \\
gender, $n$ (\%) & $6(22.22)$ & $8(27.59)$ & $2(8.00)$ \\
female & $21(77.78)$ & $21(72.41)$ & $23(92.00)$ \\
male & & \\
\hline
\end{tabular}

proaches are a standard means of conducting metabolomic analyses, as they offer a high-resolution and sensitive means of identifying metabolite profiles in complex sample types. ${ }^{10,11}$ Many studies to date have employed LC-MS-based metabolomic profiling approaches to characterize small-molecule metabolites in specific tissues, organs, and biofluids to identify biomarkers associated with specific disease states and to better understand the molecular basis of the disease-related phenotypes. Prior studies have specifically sought to identify metabolic biomarkers of HCC in patient serum, urine, plasma, and fecal samples, ${ }^{12-17}$ comparing the HCC patient samples to those from healthy controls or patients with benign liver tumors. However, whether these same biomarkers can discriminate between $\mathrm{CHB}, \mathrm{LC}$, and $\mathrm{HCC}$ patients remains to be determined, and identifying biomarkers that can reliably differentiate between these high-risk patient groups is vital.

In the present study, we used an LC/MS approach to differentiate the serum profiles of $\mathrm{CHB}, \mathrm{LC}$, and HCC patients. Based on the differential metabolites identified through this approach, we additionally identified metabolic pathways and correlation networks that may be of value for the clinical diagnosis of HCC.

\section{RESULTS AND DISCUSSION}

Metabolite Identification. We began by employing an LC-MS/MS approach to identify metabolite biomarkers of CHB, LC, and HCC, with $\mathrm{m} / z$ and RT values for each feature being collected. Quality control (QC) for these metabolic features was performed based upon the overall MS signal intensity controlled by the total ion chromatogram (TIC), $m / z$ peak width, and retention time values (Table S1). After MSConvert was used to convert raw will format data into an mzXML format [27], peak alignment and extraction was performed and peak area values were calculated. We ultimately determined that 21236 and 15665 metabolites were identified in positive- and negative-ion modes, respectively, of which 12413 and 9645, respectively, were of high quality. Of these, we were in turn able to successfully annotate 7403 and 6083 metabolites obtained in positive- and negative-ion modes, respectively (Table 2 ). The technical selection route of the diagnostic biomarker along with disease progression from $\mathrm{CHB}, \mathrm{LC}$, to HCC in the patients displayed in Figure 1 and Tables 1 and 2.

Cluster Analysis and PCA. Global metabolic differences between CHB, LC, and HCC samples were next evaluated. We began by clustering these identified metabolites into a heatmap that was able to readily differentiate between these three patient groups (Figure 2a,b). A further principal component 
Table 2. Statistics for Quantitative Metabolites

\begin{tabular}{lccc} 
mode & all metabolites & high-quality metabolites & all annotated \\
POS & 21236 & 12413 & 7406 \\
NEG & 15665 & 9645 & 6083 \\
total & 36901 & 22058 & 13489 \\
\hline
\end{tabular}

analysis (PCA) revealed a clear differentiation between these three patient groups in both positive-ion mode (Figure $2 \mathrm{c}$ ) and negative-ion mode (Figure $2 \mathrm{~d}$ ), with the variability between the samples in these PCA plots being attributable to differences in metabolite levels.

Differential Analysis. We next conducted a differential analysis of the identified high-quality metabolites to gain quantitative insights into their relative levels in our CHB, LC, and HCC patient samples. The metabolite levels were compared on the basis of both fold-change and p-vales, revealing that 279 and 307 metabolites were upregulated and downregulated, respectively, in HCC patient samples relative to LCC patient samples (Table 3 and Figure 3 ). In addition, we identified metabolites that were found to exhibit significant differences in abundance when comparing positive- and negative-ion mode data, including benzenoids, lipids and lipid-like molecules, organic acids and derivatives, organic oxygen compounds, phenylpropanoids and polyketides, and alkaloids and derivatives. In addition, we identified 858 and 1720 metabolites that were upregulated and downregulated, respectively, in $\mathrm{HCC}$ patient samples relative to $\mathrm{CHB}$ patient samples (Table 3 and Figure 4). These differentially abundant
Table 3. Statistics for Differential Metabolites between Different Groups

$\begin{array}{llrcr}\text { mode } & \text { comparison } & \text { all } & \text { up } & \text { down } \\ \text { POS } & \text { HCC vs LC } & 12413 & 112 & 125 \\ \text { POS } & \text { HCC vs CH } & 12413 & 397 & 916 \\ \text { POS } & \text { LC vs CH } & 12413 & 199 & 182 \\ \text { NEG } & \text { HCC vs LC } & 9645 & 165 & 82 \\ \text { NEG } & \text { HCC vs CH } & 9645 & 461 & 804 \\ \text { NEG } & \text { LC vs CH } & 9645 & 148 & 176\end{array}$

metabolites included benzenoids, lipids and lipid-like molecules, organic acids and derivatives, organic oxygen compounds, organoheterocyclic compound, phenylpropanoids and polyketides, and alkaloids and derivatives thereof as detected in both negative- and positive-ion modes. Lastly, we identified 347 and 329 metabolites that were upregulated and downregulated, respectively, in the LC patient samples relative to the CHB patient samples (Table 3 and Figure 5). These included benzenoids, lipids and lipid-like molecules, organic acids and derivatives, organoheterocyclic compounds, and phenylpropanoids and polyketides.

Kyoto Encyclopedia of Genes and Genomes (KEGG) Analysis. Liver disease progression and HCC development are complex processes associated with significant changes in secondary metabolite production. As such, we next employed a Kyoto Encyclopedia of Genes and Genomes (KEGG) functional enrichment approach to identify metabolic changes consistent with the differential metabolite profiles identified in
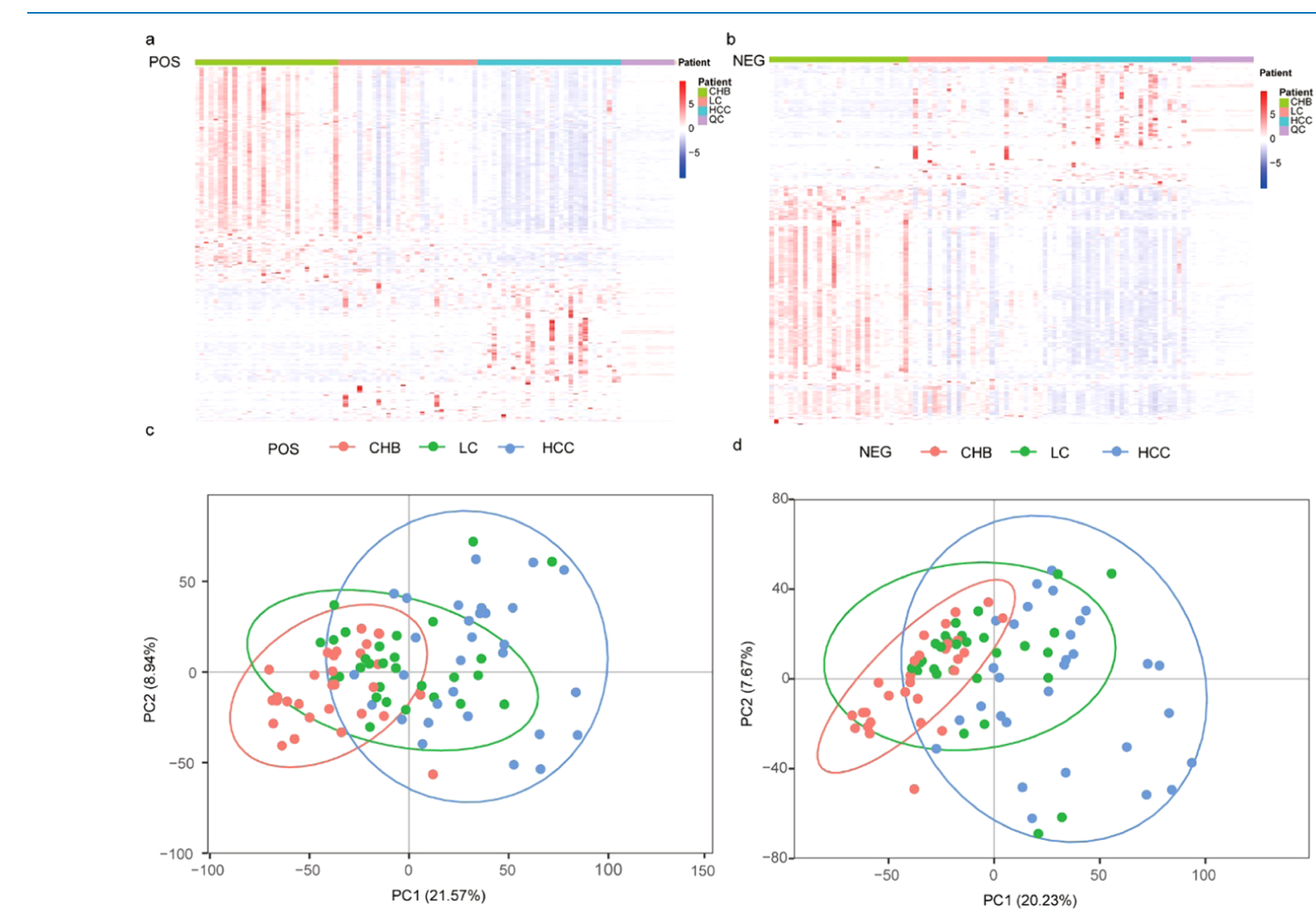

Figure 2. Heatmap of the metabolites identified among CHB, LC, and HCC patients in the (a) POS model and the (b) NEG model. The heatmap scale ranges from -5 to +5 on a $\log 2$ scale. The principal component analysis (PCA) score plots of the three truffle types in the (c) POS model and the (d) NEG model. 

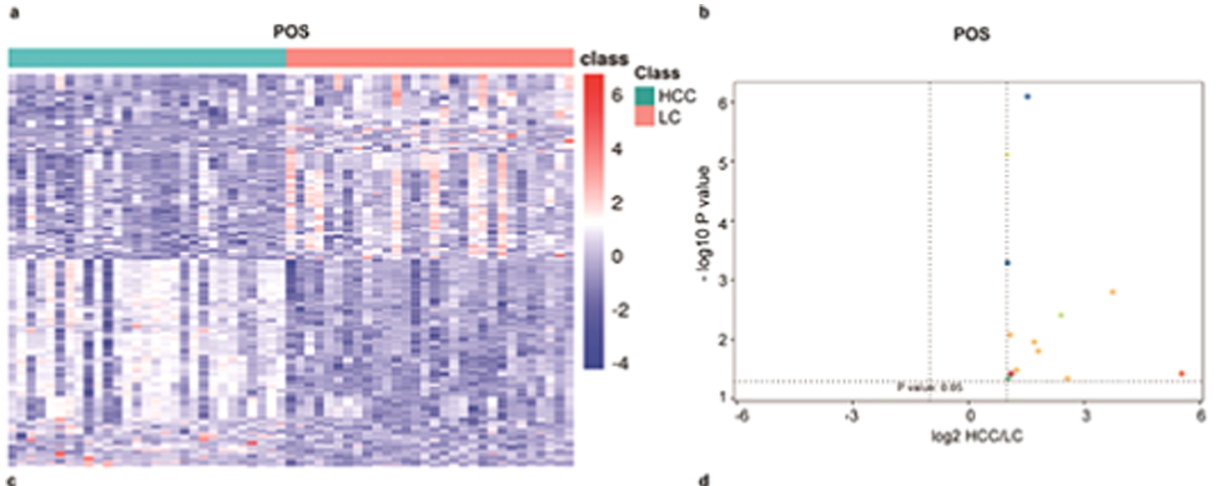

- Benzensioss

Likiss ass lipis tho molecules Organcic asids and olorvesives

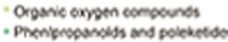
- Unionor
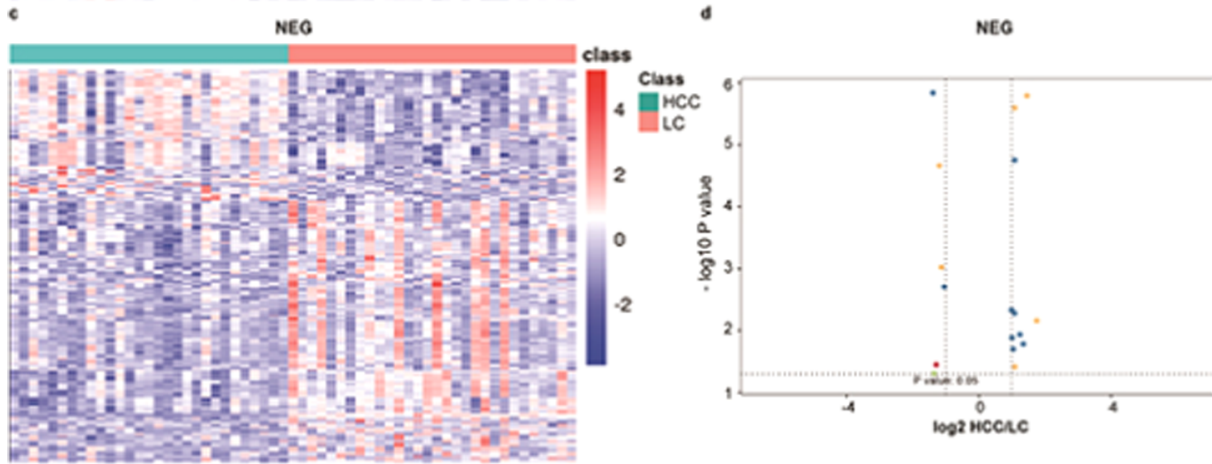

- Nrakews and derimatives

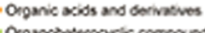

Organcheterocyolic comsounss unkexper

Figure 3. Heatmap of the different metabolites between HCC and LC patients in the (a) NEG model and the (c) POS model. The scatterplot shows the different metabolites of MS2 between HCC and LC patients in the (b) NEG model and the (d) POS model.
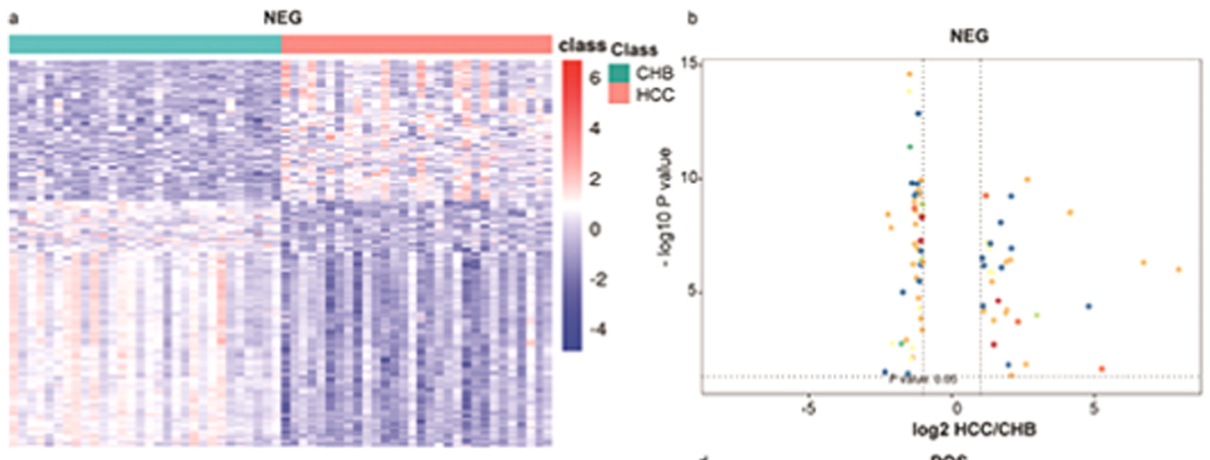

- Benzencids

"Wiats and lisid the molecters

Organic expen compounds

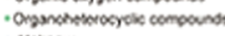
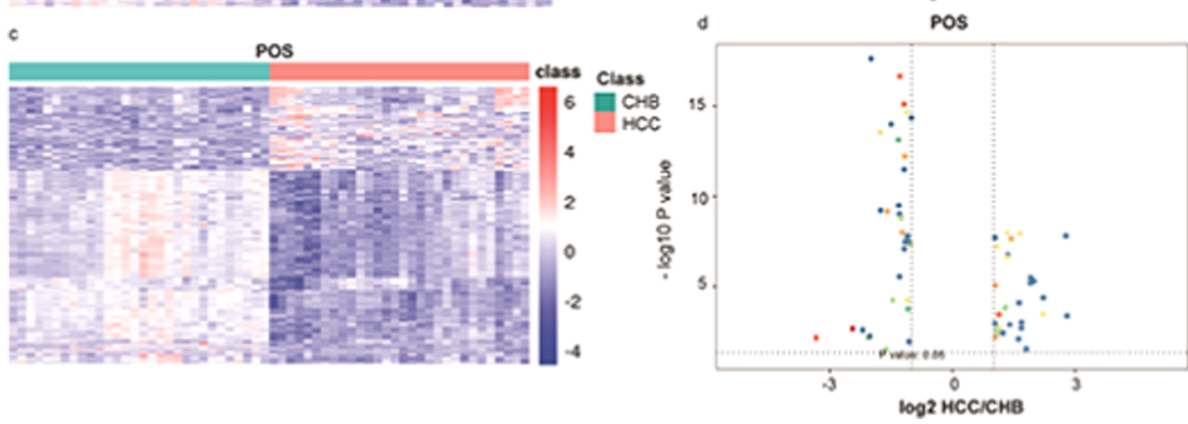

- Nhaicioss and derivetives

- Benzenceiss

Libiss and igidike molecules

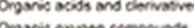

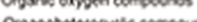

Organsheterexyile compounds

- unanow

Figure 4. Heatmap of the different metabolites between HCC and CHB patients in the (a) NEG model and the (c) POS model. The scatterplot shows the different metabolites of MS2 between HCC and CHB patients in the (b) NEG model and the (d) POS model.

our different patient serum samples. The majority of the secondary metabolites that were differentially abundant when comparing HCC and LC samples were associated with defined KEGG metabolic pathways including GABAergic synapses, pentose and glucuronate interconversion, FoxO signaling, amino sugar and nucleotide sugar metabolism, and alanine, aspartate, and glutamate metabolism (Figure S1). Metabolites that were differentially abundant between the HCC and $\mathrm{CHB}$ patient groups were found to be associated with bile secretion, pentose and glucuronate interconversion, $\mathrm{C} 5$-branched dibasic acid metabolism, D-glutamine and D-glutamate metabolism, histidine metabolism, 2-oxocarboxylic acid metabolism, and glyoxylate and dicarboxylate metabolism (Figure S2). In addition, metabolites that were differentially abundant between the LC and CHB groups were associated with steroid hormone biosynthesis, glycerophospholipid metabolism, unsaturated fatty acid biosynthesis, glycerolipid metabolism, ABC trans- 

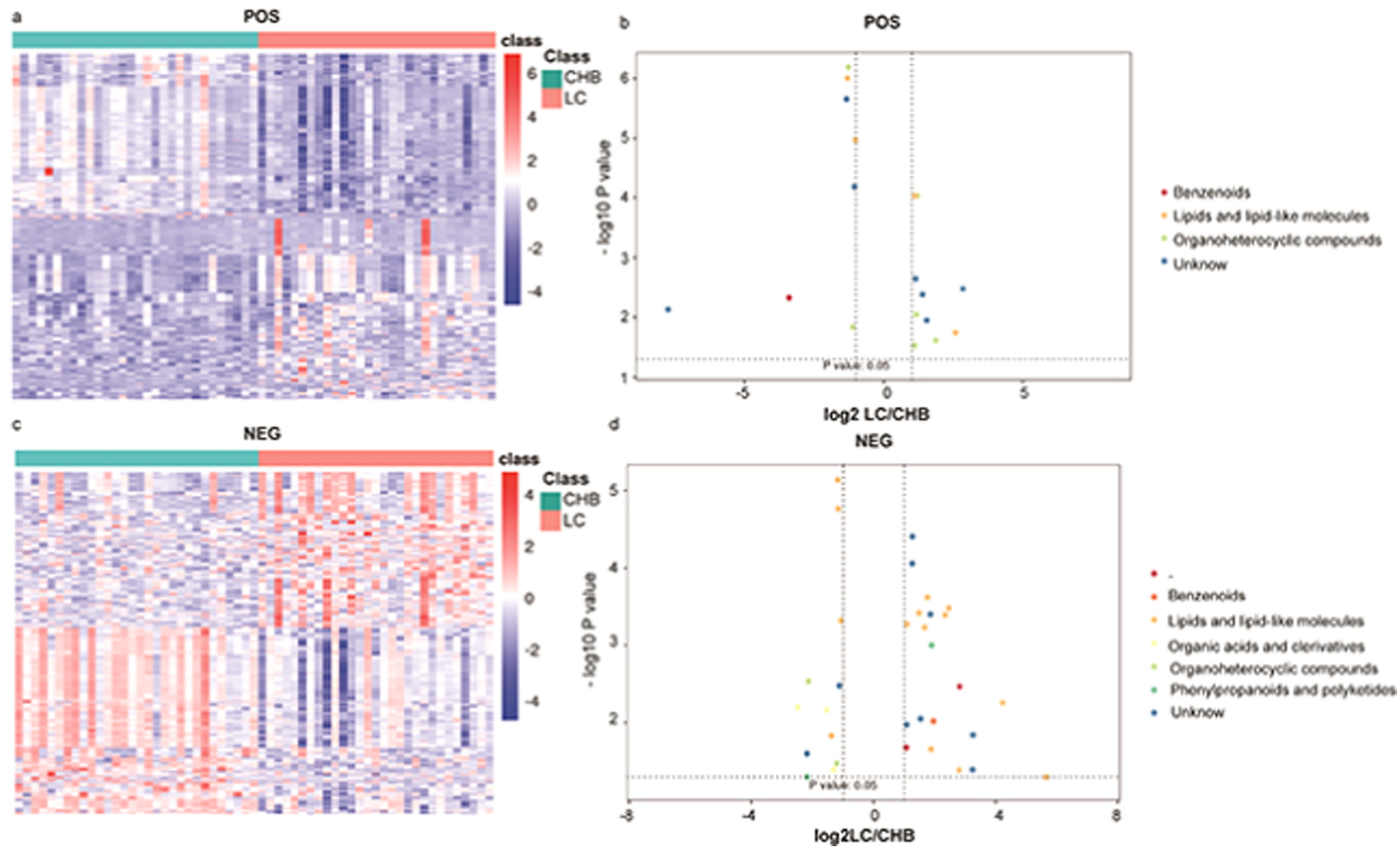

Figure 5. Heatmap of the different metabolites between LC and CHB patients in the (a) NEG model and the (c) POS model. The scatterplot shows the different metabolites of MS2 between LC and CHB patients in the (b) NEG model and the (d) POS model.
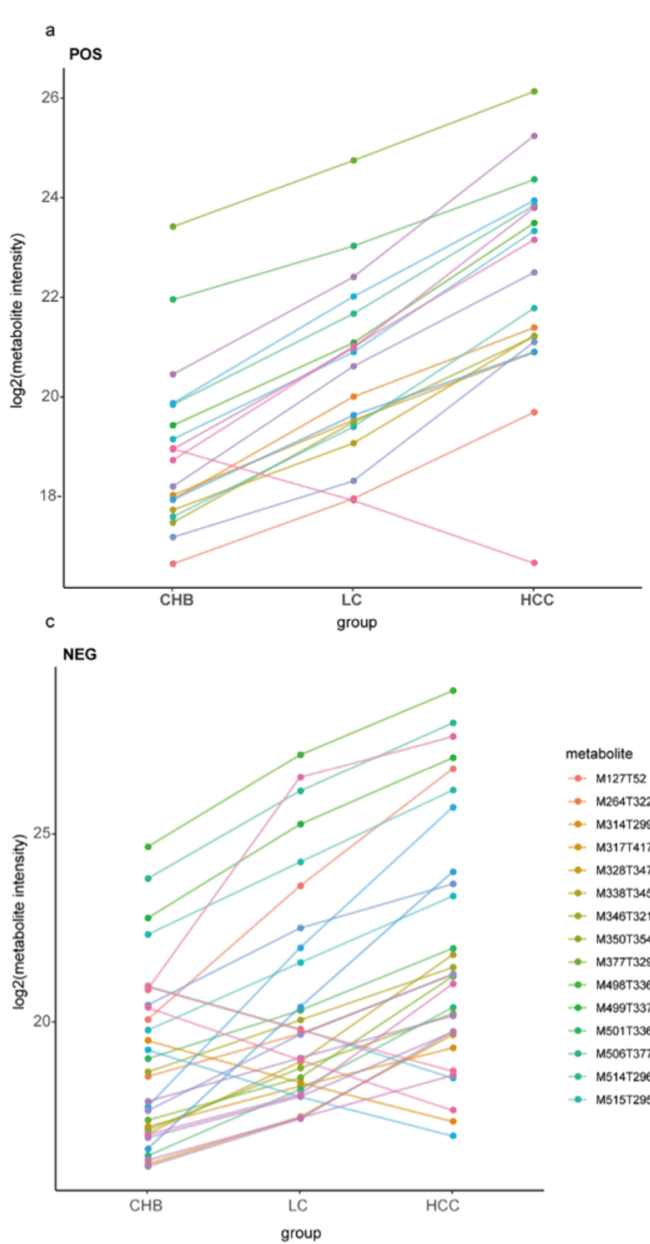

b
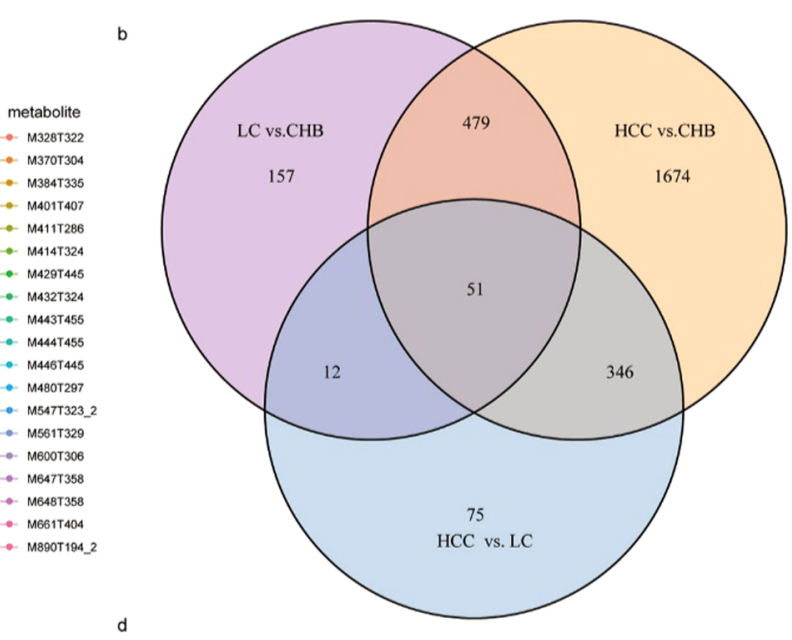

NEG

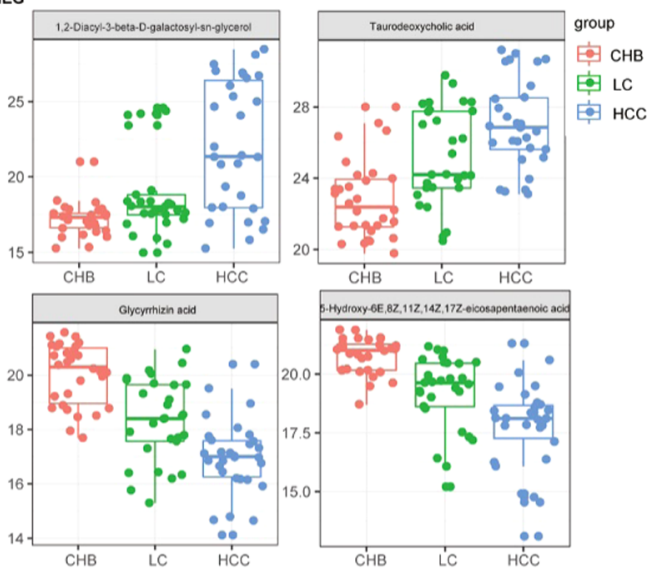

Figure 6. Identify trends in metabolic changes associated with liver disease progression from CHB to LC to HCC in the POS model (a) and the NEG model (c). Venn diagram result between the three groups, HCC vs LC, HCC vs CHB, and LC vs CHB (b). Focus key metabolic change associated with liver disease progression from CHB to LC to HCC in NEG model (d). 

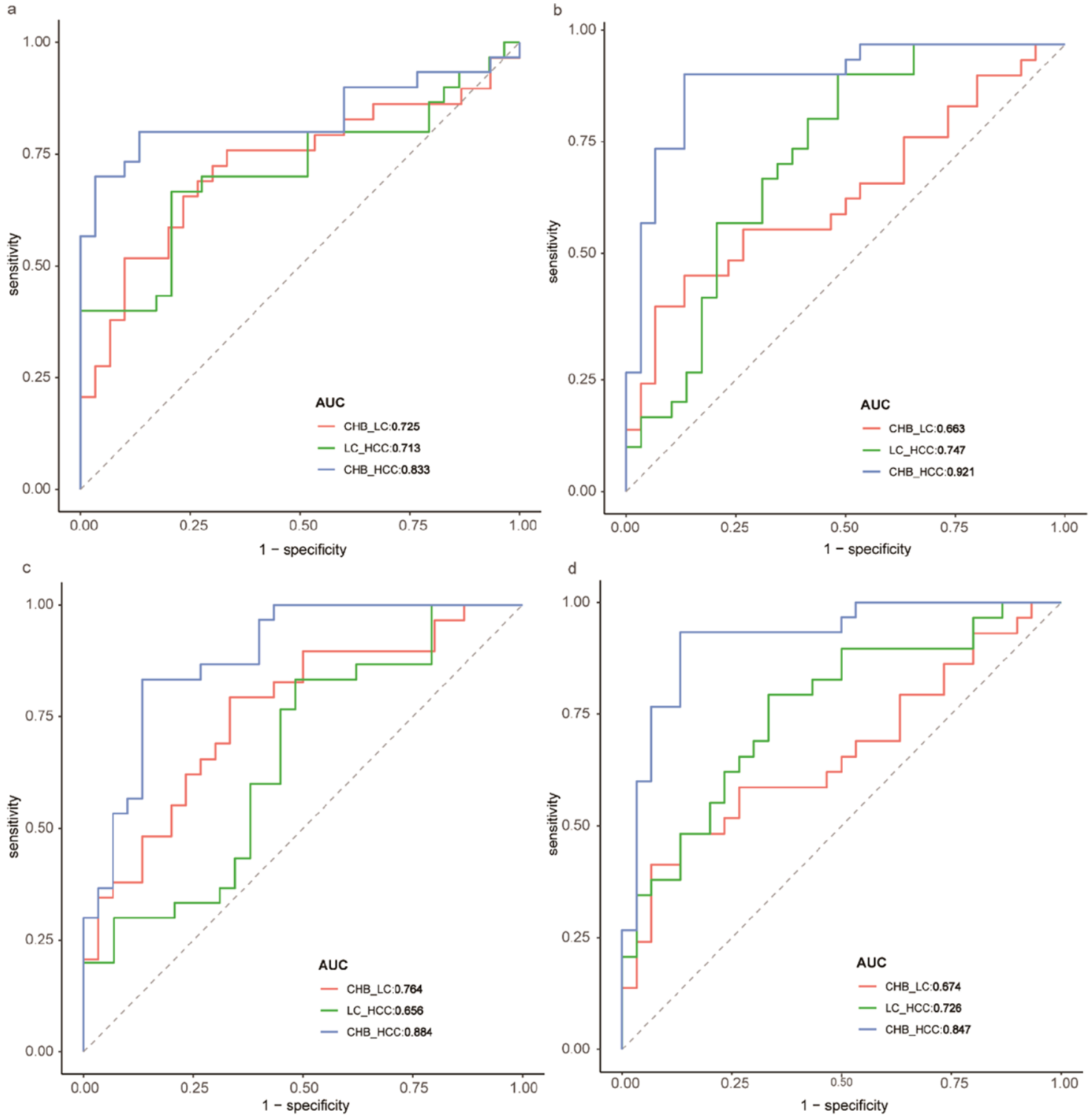

Figure 7. ROC analysis to test the prediction power of 1,2-Diacyl-3- $\beta$-D-galactosyl-sn-glycerol (a), 5-hydroxy-6E,8Z,11Z,14Z,17Z-eicosapentaenoic acid (b), taurodeoxy cholic acid (c), and glycyrrhizic acid (d), which thought the larger area under the ROC curve as a better model for predict diagnosis performance with liver disease progression.

porters, retrograde endocannabinoid signaling, and riboflavin metabolism (Figure S3).

Metabolites Associated with Progressive Liver Disease. Lastly, we evaluated metabolite profiles to identify trends in metabolic changes associated with liver disease progression from CHB to LC to HCC. We found that certain metabolites gradually decreased with disease progression, whereas others gradually increased (Figure 6a,c). A Venn diagram analysis revealed that there were 1674 different metabolites when comparing $\mathrm{HCC}$ and $\mathrm{CHB}$ samples, 157 when comparing LC and CHB samples, and 75 when comparing LC and HCC samples (Figure $6 \mathrm{~b}$ and Table S2). Overall, these findings suggest that there are relatively lower different metabolites that are associated with liver disease progression to HCC. Specifically, we found that taurodeoxy cholic acid (TCA) and 1,2-diacyl-3- $\beta$-D-galactosyl-sn-glycerol levels gradually increased with liver disease progression, whereas 5-hydroxy-6E,8Z,11Z,14Z,17Z-eicosapentaenoic acid and glycyrrhizic acid levels gradually decreased with disease progression (Figure 6d).

We performed a ROC analysis to test the prediction power of 1,2-diacyl-3- $\beta$-D-galactosyl-sn-glycerol,5-hydroxy$6 E, 8 Z, 11 Z, 14 Z, 17 Z$-eicosapentaenoic acid, taurodeoxy cholic acid (TCA), and glycyrrhizic acid, which thinked the larger area under the ROC curve as a better model for predict diagnosis performance with liver disease progression. The predictive ability of 1,2 -diacyl-3- $\beta$-D-galactosyl-sn-glycerol was higher in $\mathrm{HCC}$ vs $\mathrm{CHB}$ than $\mathrm{LC}$ vs $\mathrm{CHB}$ and $\mathrm{HCC}$ vs LC $\left(\mathrm{AUC}_{\mathrm{HCC} \text { vs } \mathrm{CHB}}=0.833\right.$ vs $\mathrm{AUC}_{\mathrm{LC} \text { vs } \mathrm{CHB}}=0.725$ vs $\mathrm{AUC}_{\mathrm{HCC} \text { vs } \mathrm{LC}}=0.713$; Figure $\left.7 \mathrm{a}\right)$. The predictive ability of 5-hydroxy-6E,8Z,11Z,14Z,17Z-eicosapentaenoic acid was higher in $\mathrm{HCC}$ vs $\mathrm{CHB}$ than $\mathrm{LC}$ vs $\mathrm{CHB}$ and $\mathrm{HCC}$ vs $\mathrm{LC}$ $\left(\mathrm{AUC}_{\mathrm{HCC} \text { vs } \mathrm{CHB}}=0.921\right.$ vs $\mathrm{AUC}_{\mathrm{LC} \text { vs } \mathrm{CHB}}=0.663$ vs $\mathrm{AUC}_{\mathrm{HCC} \text { vs } \mathrm{LC}}=0.747$; Figure $7 \mathrm{~b}$ ). The predictive ability of taurodeoxy cholic acid was higher in HCC vs CHB than LC vs $\mathrm{CHB}$ and $\mathrm{HCC}$ vs $\mathrm{LC}\left(\mathrm{AUC}_{\mathrm{HCC}}\right.$ vs $\mathrm{CHB}=0.884$ vs $\mathrm{AUC}_{\mathrm{LC} \text { vs } \mathrm{CHB}}=0.663$ vs $\mathrm{AUC}_{\mathrm{HCC} \text { vs LC }}=0.747$; Figure 7c). 

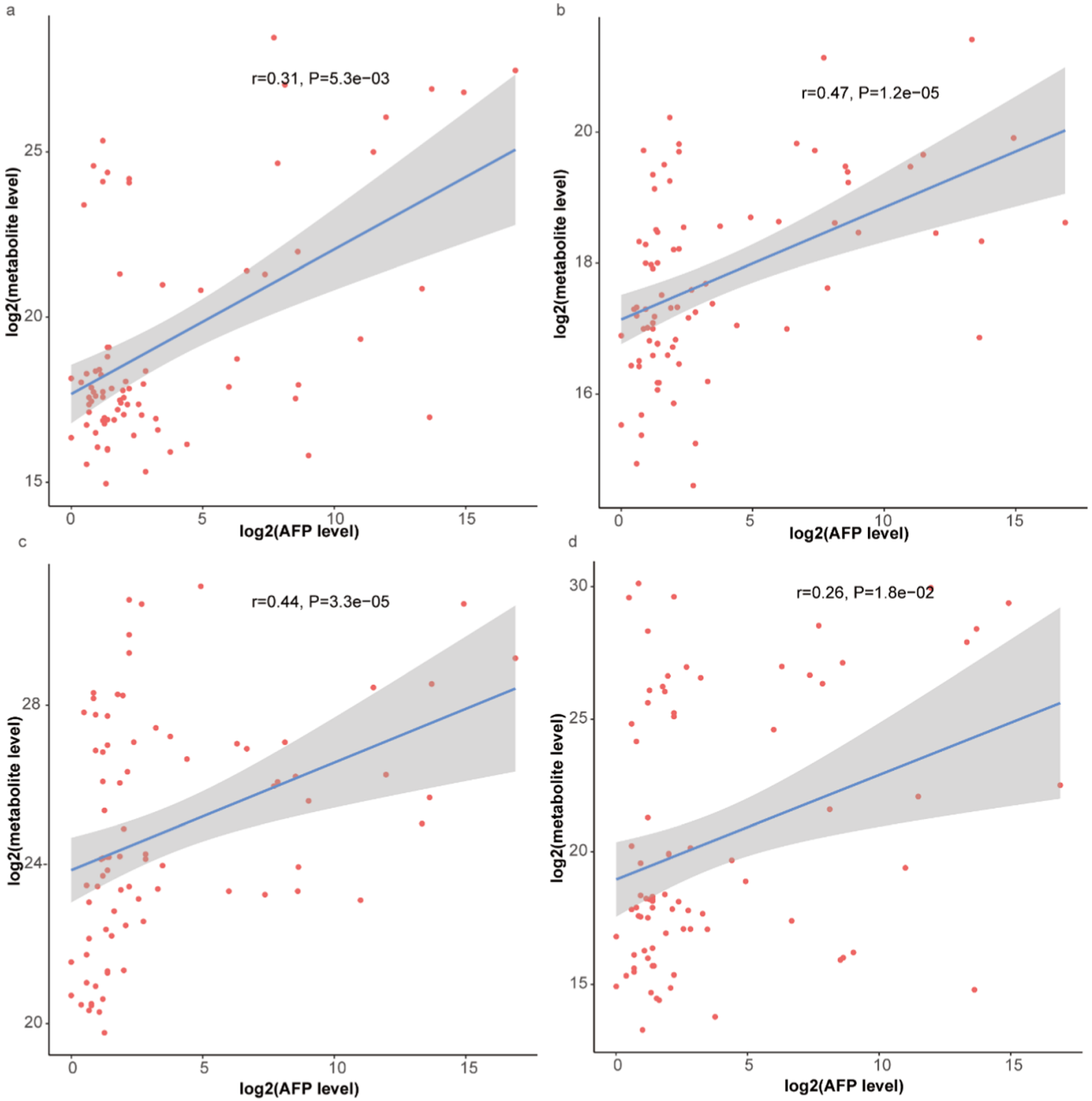

Figure 8. Correlation analysis between the levels of these different metabolites and the AFP levels: 1,2-diacyl-3- $\beta$-D-galactosyl-sn-glycerol (a); 5hydroxy-6E,8Z,11Z,14Z,17Z-eicosapentaenoic acid (b); taurodeoxy cholic acid (c); and glycyrrhizic acid (d).

The predictive ability of glycyrrhizic acid was higher in HCC vs CHB than LC vs CHB and HCC vs LC $\left(\mathrm{AUC}_{\mathrm{HCC} \text { vs } \mathrm{CHB}}=\right.$ 0.847 vs $\mathrm{AUC}_{\mathrm{LC} \text { vs } \mathrm{CHB}}=0.726$ vs $\mathrm{AUC}_{\mathrm{HCC} \text { vs } \mathrm{LC}}=0.574$; Figure $7 d$ ). The four metabolites could offer better diagnosis performance for the three-way comparisons (HCC vs LC, LC vs $\mathrm{CHB}, \mathrm{HCC}$ vs $\mathrm{CHB}$ ) using a regression model (Figure S6). These results showed that these metabolites had diagnosis performance with liver disease progression.

Correlation Analysis between the Levels of These Different Metabolites and the BMI, AFP Levels, Age. Subsequently, we have done a correlation analysis between the levels of these different metabolites and the body mass index (BMI), alpha-fetoprotein (AFP) levels, and age. The results showed that 1,2-diacyl-3- $\beta$-D-galactosyl-sn-glycerol,5-hydroxy$6 E, 8 Z, 11 Z, 14 Z, 17 Z$-eicosapentaenoic acid, taurodeoxy cholic acid (TCA), and glycyrrhizic acid have significant positive correlations with the AFP levels $(P<0.05)$. 5-Hydroxy$6 E, 8 Z, 11 Z, 14 Z, 17 Z$-eicosapentaenoic acid (Figure 8b) and taurodeoxy cholic acid (Figure 8c) had more correlations than 1,2-diacyl-3- $\beta$-D-galactosyl-sn-glycerol (Figure 8a) and glycyr- rhizic acid (Figure 8d). These four metabolites have no significant correlation with BMI (Figure S4) and have a significant correlation with age (Figure S5).

In the present study, a deep understanding of the metabolites involved in the development of HCC is needed to identify new biomarkers to diagnose HCC in the early stages and develop more effective therapeutic strategies. ${ }^{18-20}$

So, we employed an LC-MS/MS approach to identify metabolite profiles that were characteristic of $\mathrm{CHB}, \mathrm{LC}$, and HCC patient serum samples. A Venn diagram analysis revealed relatively lesser different metabolites in $\mathrm{CHH}$ vs $\mathrm{LC}$ patients compared to $\mathrm{CHB}$ vs $\mathrm{CHB}$ patients and $\mathrm{CHH}$ vs $\mathrm{LC}$ patients. TCA, glycyrrhizin acid (GA), 1,2-diacyl-3- $\beta$-D-galactosyl-snglycerol, and 5-hydroxy-6E,8Z,11Z,14Z,17Z-eicosapentaenoic acid were found to be the metabolites that were most closely associated with liver disease progression from $\mathrm{CHB}$ to $\mathrm{LC}$ to HCC. The ROC results showed that these metabolites had diagnosis performance with liver disease progression.

Bile acid biosynthesis dysfunction has been found to be linked to the onset and progression of liver cancer. ${ }^{21}$ Indeed, 
bile acids play can promote NF- $\kappa \mathrm{B}$ signaling and subsequent inflammation that can drive HCC progression. ${ }^{22-24}$ Prior studies have demonstrated that patients with hepatitis and LC exhibit altered bile acid levels, ${ }^{13,25,26}$ and there is evidence that TCA levels are elevated in the serum of $\mathrm{CHB}$ patients relative to healthy control individuals. ${ }^{25}$ The use of bile acids as biomarkers of hepatic injury has thus been proposed as a means of monitoring patients for metabolic changes associated with LC development and progression. ${ }^{26}$ In this study, we found that the TCA levels continued to increase as the disease progressed from $\mathrm{CHB}$ to $\mathrm{LC}$ to $\mathrm{HCC}$, indicating that TCA may be a viable biomarker that can be used to monitor and diagnose patients at high risk of HCC.

GA is a pentacyclic triterpenoid and an active aglycone derived from glycyrrhizin (GL). Hepatocytes express high levels of GA receptors on their cell membranes, ${ }^{27}$ and GA receptor expression has been shown to increase by 1.5- to 5fold in tumor tissues relative to control tissues. ${ }^{28}$ GA is known to exert antiapoptotic and anti-inflammatory activities owing to its ability to suppress caspase- 3 activation and tumor necrosis factor (TNF) signaling, potentially explaining the hepatoprotective properties of this compound. ${ }^{29} \mathrm{~A}$ number of recent studies have explored the use of GA-modified liposomes to improve hepatic drug delivery for the treatment of HCC, ${ }^{30-32}$ and randomized clinical trials have provided evidence that GL can protect against hepatic damage in CHB and LC patients, promoting the restoration of liver function. ${ }^{33}$ To date, however, few studies have examined how GA levels are associated with liver disease progression. Our results suggest that GA levels declined with the progression from $\mathrm{CHB}$ to $\mathrm{LC}$ to HCC, suggesting that the GA levels may be a valuable biomarker that can help to diagnose HCC onset in LC patients.

Eicosapentaenoic acid (EPA) is a bioactive omega-3 polyunsaturated fatty acid that is found in high levels in fish oil and exhibits diverse anti-inflammatory and antilipogenic properties. $^{34}$ EPA has been found to inhibit obesity-related HCC development, functioning via inhibiting the activation of the STAT3 transcription factor and subsequent production of the pro-oncogenic inflammatory transcription factor IL- $6 .{ }^{35}$ In a separate study, $\mathrm{Lu}$ et al. determined that EPA levels were significantly lower in CHB, LC, and HCC patients. ${ }^{36}$ Our results are consistent with these findings, suggesting that 5hydroxy- $6 E, 8 Z, 11 Z, 14 Z, 17 Z$-eicosapentaenoic acid can be used as a stable biomarker of LC and HCC in CHB patients. We also found that 1,2-diacyl-3- $\beta$-D-galactosyl-sn-glycerol levels declined progressively with the advancement of liver disease in these patients. This metabolite is associated with glycerolipid metabolism, but its relevance in the context of $\mathrm{CHB}, \mathrm{LC}$, and HCC remains to be characterized.

Wang et al. and Cho et al. have searched for biomarkers to differentiate early-stage hepatocellular carcinoma from cirrhosis or healthy control. ${ }^{37,38}$ Few studies have screened diagnostic biomarker with disease progression from $\mathrm{CHB}, \mathrm{LC}$, to HCC in the patients.

We characterized changes in serum metabolite profiles with liver disease progression from CHB to LC to HCC via an LCMS/MS-based approach. Therefore, our study was innovative in the selection of patients. We identified those metabolites that best differentiated HCC patients from LC patients based upon these analyses, annotating them via comparing identified monoisotopic ion masses to mass-based reference databases. We ultimately identified TCA and 1,2-diacyl-3- $\beta$-D-galactosyl- $s n$-glycerol levels as having increased gradually with liver disease progression from CHB to LC to HCC, whereas levels of GA and 5-hydroxy-6E,8Z,11Z,14Z,17Z-eicosapentaenoic acid decreased with disease progression. These metabolites are thus promising biomarkers that have the potential to be used to detect early-stage HCC and monitor disease progression in high-risk patients.

\section{METHODS}

Study Participant Characteristics. Baseline patient characteristics for individuals enrolled in this study are compiled in Table 1 . In total, we included samples from 30 $\mathrm{CHB}$ patients (mean age: 42; 76.7\% male), 29 LC patients (mean age: $52 ; 68.7 \%$ male), and $30 \mathrm{HCC}$ patients (mean age: 58; $86.7 \%$ male).

Sample Preparation. The serum samples from these patients were thawed on ice, and a 50\% methanol solution was then used for metabolite extraction. Briefly, a $20 \mu \mathrm{L}$ volume of each sample was then mixed with $120 \mu \mathrm{L}$ of cold $50 \%$ methanol for $1 \mathrm{~min}$, incubated for $10 \mathrm{~min}$ at room temperature for $10 \mathrm{~min}$, and then allowed to rest overnight at $-20{ }^{\circ} \mathrm{C}$. The samples were then spun for $20 \mathrm{~min}$ at $4000 \mathrm{~g}$, and supernatants were isolated and stored at $-80{ }^{\circ} \mathrm{C}$ in 96 -well plates for LCMS analyses. In addition, $10 \mu \mathrm{L}$ from each extract was pooled to prepare a quality control (QC) solution.

LC-MS/MS Analysis. A DIONEX Ultimate 3000 HPLC system (Thermo Fisher Scientific) was used for LC-MS/MS metabolomic analyses. Briefly, $10 \mu \mathrm{L}$ per sample was injected into a Synergi $4 \mu \mathrm{m}$ Hydro-RP $80 \AA \AA, 250 \mathrm{~mm} \times 3.0 \mathrm{~mm}$ column (Phenomenex, Le Pecq, France). Mobile phases for this analysis included $0.1 \%$ formic acid in water (A) and $0.1 \%$ formic acid in acetonitrile (B). Gradient settings were as follows: $0-5 \mathrm{~min}, 0 \% \mathrm{~B}$; 5-21 $\mathrm{min}, 0-95 \% \mathrm{~B} ; 21-21.5 \mathrm{~min}$, $95 \% \mathrm{~B} ; 21.5-22 \mathrm{~min}, 95-0 \% \mathrm{~B}$; and $22-25 \mathrm{~min}, 0 \% \mathrm{~B}$. The flow rate was maintained at $0.9 \mathrm{~mL} / \mathrm{min}$. A Q Exactive Plus Orbitrap mass spectrometer (Thermo Scientific) with a heated electrospray ionization source (HESI II) was utilized in negative- and positive-ion modes for MS analyses. Highresolution accurate-mass full-scan MS and top 5 MS2 spectra were collected in a data-dependent acquisition mode at a respective resolving power of 70000 and 35000 at $\mathrm{m} / z 400$. In addition, the QC samples were injected at the start of each run and as every 10th sample to ensure the stability of these MS analyses.

Metabolomic Profiling. MSConvert (v2.1, ProteoWizard) was used to convert raw data into the mzXML format, ${ }^{39}$ after which MZmine (v2.29) was used to separately analyze the data acquired in negative- and positive-ion modes. ${ }^{40} \mathrm{~A}$ noise threshold of 105 was used when constructing isolated chromatograms for each mass, after which validated peaks were selected using a local minimum search algorithm. A random sample consensus (RANSAC) algorithm was then used to align peaks with a $10 \mathrm{ppm} \mathrm{m} / z$ tolerance and a $1 \mathrm{~min}$ retention time tolerance. The missing values were replaced with those corresponding to those within the same $\mathrm{m} / z$ and $\mathrm{RT}$ ranges when possible, and only peaks lacking missing values after gap-filling were retained for subsequent analyses. The human metabolome database (HMDB, v3.0) ${ }^{41}$ was then used for peak identification, with a 15 ppm mass tolerance. We then individually verified the identities of selected metabolites with a VIP >3 based upon MS and MS2 spectra, with only identified metabolites being retained for downstream analyses. We then combined results from positive- and negative-ion 
modes and retained metabolites with higher intensity mean values.

Data Analysis. XCMS software was used for peak picking, peak grouping, retention time correction, second peak grouping, and isotope and adduct annotation. R XCMS, CAMERA, and metaX tools were used to process mzXMLformatted data files. Ions were identified based upon RT and $m / z$ data, with peak intensities being recorded and used to generate a three-dimensional matrix that incorporated sample names, provided ion intensity information, and arbitrarily assigned peak indices (retention time $-m / z$ pairs). These metabolites were then annotated using the KEGG and HMDB databases via exact matching of the sample $m / z$ data to those in these databases. When mass differences between the observed and predicted values were $<10 \mathrm{ppm}$, the metabolites were annotated and subjected to further validation based upon the isotopic distribution measurements. Metabolite identification was then validated using an in-house fragment spectrum metabolite library, and metaX was then used to further preprocess peak intensity data. Features that were present in $<50 \%$ of the QC samples of $<80 \%$ of the biological samples were not retained for analysis, while a $k$-nearest-neighbor algorithm was used to impute missing values for the remaining peaks to improve the overall data quality. Outliers and batch effects were next assessed via a PCA approach, while the QC data was fitted to QC-based robust LOESS signal correction based upon the order of injection in an effort to minimize any drift in the signal intensity values over time. Relative standard deviations for metabolites were also calculated for all QC samples, and any that had $>30 \%$ standard deviation was removed. Differences in metabolite levels between two sample groups were compared using Student's $t$ tests, with $P$-values being adjusted using the Benjamini-Hochberg method to control for multiple testing. In addition, metaX was used for supervised PLS-DA to identify metabolites capable of discriminating between groups, with VIP values being determined. A VIP of $\geq 1.0$ was used for important feature selection.

\section{ASSOCIATED CONTENT}

\section{SI Supporting Information}

The Supporting Information is available free of charge at https://pubs.acs.org/doi/10.1021/acsomega.0c04259.

Identified metabolites classified into the top 20 KEGG pathways with HCC vs LC patients in the NEG model (Figure S1); identified metabolites classified into the top 20 KEGG pathways with $\mathrm{HCC}$ vs $\mathrm{CHB}$ patients in the POS model (a) and the NEG model (b) (Figure S2); identified metabolites classified into the top 20 KEGG pathways with LC vs CHB patients in the POS model (a) and the NEG model (b) (Figure 3S); correlation analysis between the levels of these different metabolites and the BMI levels (Figure S4); correlation analysis between the levels of these different metabolites and the age (Figure S5); and the four metabolites could offer better AUC for the three-way comparisons (HCC vs LC, $\mathrm{LC}$ vs $\mathrm{CHB}, \mathrm{HCC}$ vs $\mathrm{CHB}$ ) using a regression model (Figure S6) (PDF)

Ion intensity information and retention time of the metabolites (Table S1) (XLSX)

Different metabolites between three groups, HCC vs LC, HCC vs CHB, and LC (Table S2) (XLSX)

\section{AUTHOR INFORMATION}

\section{Corresponding Authors}

Hai J. Huang - Department of Infectious Diseases, Zhejiang Provincial People's Hospital, People's Hospital of Hangzhou Medical College, Hangzhou, Zhejiang 310014, China; Email: huanghaijun0826@163.com

Yong X. Tong - Department of Infectious Diseases, Zhejiang Provincial People's Hospital, People's Hospital of Hangzhou Medical College, Hangzhou, Zhejiang 310014, China; (i) orcid.org/0000-0003-0313-4402;

Email: tongyongxi123@sina.com

\section{Authors}

Hong Y. Pan - Department of Infectious Diseases, Zhejiang Provincial People's Hospital, People's Hospital of Hangzhou Medical College, Hangzhou, Zhejiang 310014, China

Qing Q. Wu - Department of Infectious Diseases, Zhejiang Provincial People's Hospital, People's Hospital of Hangzhou Medical College, Hangzhou, Zhejiang 310014, China; The Second Clinical Medical College, Zhejiang Chinese Medical University, Hangzhou, Zhejiang 310053, China

Qiao Q. Yin - Department of Infectious Diseases, Zhejiang Provincial People's Hospital, People's Hospital of Hangzhou Medical College, Hangzhou, Zhejiang 310014, China; Bengbu Medical College, Bengbu, Anhui 233030, China

Yi N. Dai - Department of Infectious Diseases, Zhejiang Provincial People's Hospital, People's Hospital of Hangzhou Medical College, Hangzhou, Zhejiang 310014, China; (1) orcid.org/0000-0002-7735-1381

Yi C. Huang - Department of Infectious Diseases, Zhejiang Provincial People's Hospital, People's Hospital of Hangzhou Medical College, Hangzhou, Zhejiang 310014, China

Wei Zheng - Department of Infectious Diseases, Zhejiang Provincial People's Hospital, People's Hospital of Hangzhou Medical College, Hangzhou, Zhejiang 310014, China

Tian C. Hui - Department of Infectious Diseases, Zhejiang Provincial People's Hospital, People's Hospital of Hangzhou Medical College, Hangzhou, Zhejiang 310014, China; Bengbu Medical College, Bengbu, Anhui 233030, China

Mei J. Chen - Department of Infectious Diseases, Zhejiang Provincial People's Hospital, People's Hospital of Hangzhou Medical College, Hangzhou, Zhejiang 310014, China

Ming S. Wang - Department of Infectious Diseases, Zhejiang Provincial People's Hospital, People's Hospital of Hangzhou Medical College, Hangzhou, Zhejiang 310014, China

Jia J. Zhang - Department of Infectious Diseases, Zhejiang Provincial People's Hospital, People's Hospital of Hangzhou Medical College, Hangzhou, Zhejiang 310014, China

Complete contact information is available at:

https://pubs.acs.org/10.1021/acsomega.0c04259

\section{Author Contributions}

"H.Y.P., Q.Q.W., and T.C.H. contributed equally to this work. Notes

The authors declare no competing financial interest.

\section{ACKNOWLEDGMENTS}

The study was supported by a grant from the National Science and Technology Major Project of the Ministry of Science and Technology of China (No. 2018ZX10302205-002). The authors state that there are no conflicts of interest to disclose. 


\section{REFERENCES}

(1) Kassahun, W. T.; Fangmann, J.; Harms, J.; Hauss, J.; Bartels, M. Liver resection and transplantation in the management of hepatocellular carcinoma: a review. Exp. Clin. Transplant. 2006, 4, 549-558.

(2) El-Serag, H. B.; Rudolph, K. L. Hepatocellular carcinoma: epidemiology and molecular carcinogenesis. Gastroenterology 2007, 132, 2557-2576.

(3) El-Serag, H. B.; Mason, A. C. Rising incidence of hepatocellular carcinoma in the United States. N. Engl. J. Med. 1999, 340, 745-750.

(4) Anthony, P. P. Hepatocellular carcinoma: an overview. Histopathology 2001, 39, 109-118.

(5) Onodera, H.; Ukai, K.; Minami, Y. Hepatocellular carcinoma cases with five-year survival and prognostic factors affecting the survival time. Tohoku J. Exp. Med. 1995, 176, 203-211.

(6) Thein, H. H.; Khoo, E.; Campitelli, M. A.; Zaheen, A.; Yi, Q.; De, P.; Earle, C. C. Trends in relative survival in patients with a diagnosis of hepatocellular carcinoma in Ontario: a population-based retrospective cohort study. CMAJ Open 2015, 3, E208-16.

(7) Bittermann, T.; Hoteit, M. A.; Abt, P. L.; Forde, K. A.; Goldberg, D. Waiting time and explant pathology in transplant recipients with hepatocellular carcinoma: a novel study using national data. Am. J. Transplant. 2014, 14, 1657-1663.

(8) Nicholson, J. K.; Lindon, J. C.; Holmes, E. 'Metabonomics': understanding the metabolic responses of living systems to pathophysiological stimuli via multivariate statistical analysis of biological NMR spectroscopic data. Xenobiotica 1999, 29, 11811189.

(9) Fiehn, O. Metabolomics-the link between genotypes and phenotypes. Plant Mol. Biol. 2002, 48, 155-171.

(10) Xiao, J. F.; Zhou, B.; Ressom, H. W. Metabolite identification and quantitation in LC-MS/MS-based metabolomics. TrAC, Trends Anal. Chem. 2012, 32, 1-14.

(11) Gika, H.; Theodoridis, G. Sample preparation prior to the LCMS-based metabolomics/metabonomics of blood-derived samples. Bioanalysis 2011, 3, 1647-1661.

(12) Shariff, M. I.; Ladep, N. G.; Cox, I. J.; Williams, H. R.; Okeke, E.; Malu, A.; Thillainayagam, A. V.; Crossey, M. M.; Khan, S. A.; Thomas, H. C.; Taylor-Robinson, S. D. Characterization of urinary biomarkers of hepatocellular carcinoma using magnetic resonance spectroscopy in a Nigerian population. J. Proteome Res. 2010, 9, 1096-1103.

(13) Chen, T.; Xie, G.; Wang, X.; Fan, J.; Qiu, Y.; Zheng, X.; Qi, X.; Cao, Y.; Su, M.; Wang, X.; Xu, L. X.; Yen, Y.; Liu, P.; Jia, W. Serum and urine metabolite profiling reveals potential biomarkers of human hepatocellular carcinoma. Mol. Cell. Proteomics 2011, 10, No. M110.004945.

(14) Correction: Trends in relative survival in patients with a diagnosis of hepatocellular carcinoma in Ontario: a population-based retrospective cohort study. CMAJ Open 2015, 3, E359. DOI: $10.9778 / \mathrm{cmajo} .20150109$.

(15) Cao, H.; Huang, H.; Xu, W.; Chen, D.; Yu, J.; Li, J.; Li, L. Fecal metabolome profiling of liver cirrhosis and hepatocellular carcinoma patients by ultra performance liquid chromatography-mass spectrometry. Anal. Chim. Acta 2011, 691, 68-75.

(16) Patterson, A. D.; Maurhofer, O.; Beyoglu, D.; Lanz, C.; Krausz, K. W.; Pabst, T.; Gonzalez, F. J.; Dufour, J. F.; Idle, J. R. Aberrant lipid metabolism in hepatocellular carcinoma revealed by plasma metabolomics and lipid profiling. Cancer Res. 2011, 71, 6590-6600.

(17) Gao, H.; Lu, Q.; Liu, X.; Cong, H.; Zhao, L.; Wang, H.; Lin, D. Application of $1 \mathrm{H}$ NMR-based metabonomics in the study of metabolic profiling of human hepatocellular carcinoma and liver cirrhosis. Cancer Sci. 2009, 100, 782-785.

(18) Zhu, K.; Zhan, H.; Peng, Y.; Yang, L.; Gao, Q.; Jia, H.; Dai, Z.; Tang, Z.; Fan, J.; Zhou, J. Plasma hsa_circ_0027089 is a diagnostic biomarker for hepatitis B virus-related hepatocellular carcinoma. Carcinogenesis 2020, 41, 296-302.

(19) Rao, B. C.; Lou, J. M.; Wang, W. J.; Li, A.; Cui, G. Y.; Yu, Z. J.; Ren, Z. G. Human microbiome is a diagnostic biomarker in hepatocellular carcinoma. Hepatobiliary Pancreatic Dis. Int. 2020, 19, 109-115.

(20) Cho, H. J.; Eun, J. W.; Baek, G. O.; Seo, C. W.; Ahn, H. R.; Kim, S. S.; Cho, S. W.; Cheong, J. Y. Serum Exosomal MicroRNA, miR-10b-5p, as a Potential Diagnostic Biomarker for Early-Stage Hepatocellular Carcinoma. J. Clin. Med. 2020, 9, 281.

(21) Manuel-Vázquez, A.; Latorre-Fragua, R.; Ramiro-Perez, C.; Lopez-Marcano, A.; la Plaza-Llamas, R.; Ramia, J. M. Laparoscopic gastrojejunostomy for gastric outlet obstruction in patients with unresectable hepatopancreatobiliary cancers: A personal series and systematic review of the literature. World J. Gastroenterol. 2018, 24, $1978-1988$

(22) Sun, L.; Beggs, K.; Borude, P.; Edwards, G.; Bhushan, B.; Walesky, C.; Roy, N.; Manley, M. W., Jr.; Gunewardena, S.; O’Neil, M.; Li, H.; Apte, U. Bile acids promote diethylnitrosamine-induced hepatocellular carcinoma via increased inflammatory signaling. Am. J. Physiol.: Gastrointest. Liver Physiol. 2016, 311, G91-G104.

(23) Walesky, C.; Edwards, G.; Borude, P.; Gunewardena, S.; O’Neil, M.; Yoo, B.; Apte, U. Hepatocyte nuclear factor 4 alpha deletion promotes diethylnitrosamine-induced hepatocellular carcinoma in rodents. Hepatology 2013, 57, 2480-2490.

(24) Jia, B.; Jeon, C. O. Promotion and induction of liver cancer by gut microbiome-mediated modulation of bile acids. PLoS Pathog. 2019, 15, No. e1007954.

(25) Yang, J.; Zhao, X.; Liu, X.; Wang, C.; Gao, P.; Wang, J.; Li, L.; $\mathrm{Gu}$, J.; Yang, S.; Xu, G. High performance liquid chromatographymass spectrometry for metabonomics: potential biomarkers for acute deterioration of liver function in chronic hepatitis B. J. Proteome Res. 2006, 5, 554-561.

(26) Yin, P.; Wan, D.; Zhao, C.; Chen, J.; Zhao, X.; Wang, W.; Lu, X.; Yang, S.; Gu, J.; Xu, G. A metabonomic study of hepatitis Binduced liver cirrhosis and hepatocellular carcinoma by using RP-LC and HILIC coupled with mass spectrometry. Mol. BioSyst. 2009, 5, $868-876$.

(27) Negishi, M.; Irie, A.; Nagata, N.; Ichikawa, A. Specific binding of glycyrrhetinic acid to the rat liver membrane. Biochim. Biophys. Acta, Biomembr. 1991, 1066, 77-82.

(28) Chen, J.; Jiang, H.; Wu, Y.; Li, Y.; Gao, Y. A novel glycyrrhetinic acid-modified oxaliplatin liposome for liver-targeting and in vitro/vivo evaluation. Drug Des., Dev. Ther. 2015, 9, 22652275.

(29) Li, J. Y.; Cao, H. Y.; Liu, P.; Cheng, G. H.; Sun, M. Y. Glycyrrhizic acid in the treatment of liver diseases: literature review. BioMed Res. Int. 2014, 2014, No. 872139.

(30) Yamaguchi, H.; Yu, T.; Kidachi, Y.; Akitaya, T.; Yoshida, K.; Kamiie, K.; Noshita, T.; Umetsu, H.; Ryoyama, K. Selective toxicity of glycyrrhetinic acid against tumorigenic $\mathrm{r} / \mathrm{m}$ HM-SFME-1 cells is potentially attributed to downregulation of glutathione. Biochimie 2011, 93, 1172-1178.

(31) Darvishi, B.; Manoochehri, S.; Esfandyari-Manesh, M.; Samadi, N.; Amini, M.; Atyabi, F.; Dinarvand, R. Enhanced Cellular Cytotoxicity and Antibacterial Activity of 18-beta-Glycyrrhetinic Acid by Albumin-conjugated PLGA Nanoparticles. Drug Res. 2015, $65,617-623$

(32) Tian, J.; Wang, L.; Wang, L.; Ke, X. A wogonin-loaded glycyrrhetinic acid-modified liposome for hepatic targeting with antitumor effects. Drug Delivery 2014, 21, 553-559.

(33) Matsuo, K.; Takenaka, K.; Shimomura, H.; Fujii, N.; Shinagawa, K.; Kiura, K.; Harada, M. Lamivudine and glycyrrhizin for treatment of chemotherapy-induced hepatitis B virus (HBV) hepatitis in a chronic HBV carrier with non-Hodgkin lymphoma. Leuk. Lymphoma 2001, 41, 191-195.

(34) Calder, P. C. Omega-3 polyunsaturated fatty acids and inflammatory processes: nutrition or pharmacology? Br. J. Clin. Pharmacol. 2013, 75, 645-662.

(35) Inoue-Yamauchi, A.; Itagaki, H.; Oda, H. Eicosapentaenoic acid attenuates obesity-related hepatocellular carcinogenesis. Carcinogenesis 2018, 39, 28-35. 
(36) Lu, Y.; Fang, J.; Zou, L.; Cui, L.; Liang, X.; Lim, S. G.; Dan, Y. Y.; Ong, C. N. Omega-6-derived oxylipin changes in serum of patients with hepatitis B virus-related liver diseases. Metabolomics 2018, 14, No. 26.

(37) Kim, D. J.; Cho, E. J.; Yu, K. S.; Jang, I. J.; Yoon, J. H.; Park, T.; Cho, J. Y. Comprehensive Metabolomic Search for Biomarkers to Differentiate Early Stage Hepatocellular Carcinoma from Cirrhosis. Cancers 2019, 11, 1497.

(38) Xie, J.; Zhang, A.; Wang, X. Metabolomic applications in hepatocellular carcinoma: toward the exploration of therapeutics and diagnosis through small molecules. RSC Adv. 2017, 7, 17217-17226.

(39) Holman, J. D.; Tabb, D. L.; Mallick, P. Employing ProteoWizard to Convert Raw Mass Spectrometry Data. Curr. Protoc. Bioinf. 2014, 46, 241-9.

(40) Pluskal, T.; Castillo, S.; Villar-Briones, A.; Oresic, M. MZmine 2: modular framework for processing, visualizing, and analyzing mass spectrometry-based molecular profile data. BMC Bioinf. 2010, 11, No. 395.

(41) Wishart, D. S.; Feunang, Y. D.; Marcu, A.; Guo, A. C.; Liang, K.; Vazquez-Fresno, R.; Sajed, T.; Johnson, D.; Li, C.; Karu, N.; Sayeeda, Z.; Lo, E.; Assempour, N.; Berjanskii, M.; Singhal, S.; Arndt, D.; Liang, Y.; Badran, H.; Grant, J.; Serra-Cayuela, A.; Liu, Y.; Mandal, R.; Neveu, V.; Pon, A.; Knox, C.; Wilson, M.; Manach, C.; Scalbert, A. HMDB 4.0: the human metabolome database for 2018. Nucleic Acids Res. 2018, 46, D608-D617. 